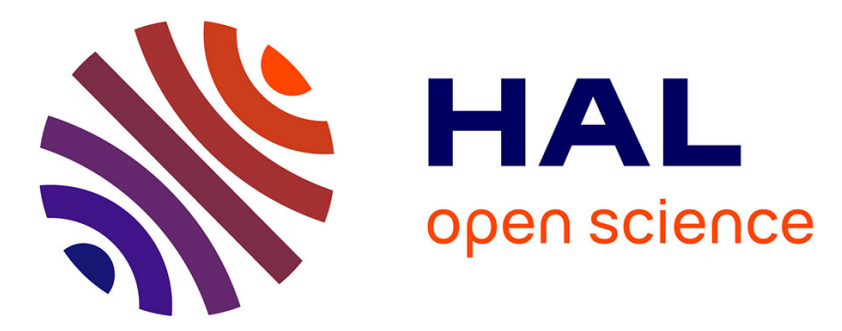

\title{
Kerr optical frequency comb generation in strontium fluoride whispering-gallery mode resonators with billion quality factor
}

Remi Henriet, Guoping Lin, Aurélien Coillet, Maxime Jacquot, Luca Furfaro, Laurent Larger, Kouomou Yanne Chembo

\section{To cite this version:}

Remi Henriet, Guoping Lin, Aurélien Coillet, Maxime Jacquot, Luca Furfaro, et al.. Kerr optical frequency comb generation in strontium fluoride whispering-gallery mode resonators with billion quality factor. Optics Letters, 2015, 40 (7), pp.1567-1570. 10.1364/OL.40.001567 . hal-02300373

\section{HAL Id: hal-02300373 \\ https://hal.science/hal-02300373}

Submitted on 20 May 2021

HAL is a multi-disciplinary open access archive for the deposit and dissemination of scientific research documents, whether they are published or not. The documents may come from teaching and research institutions in France or abroad, or from public or private research centers.
L'archive ouverte pluridisciplinaire HAL, est destinée au dépôt et à la diffusion de documents scientifiques de niveau recherche, publiés ou non, émanant des établissements d'enseignement et de recherche français ou étrangers, des laboratoires publics ou privés. 


\title{
Kerr optical frequency comb generation in strontium fluoride whispering-gallery mode resonators with billion quality factor
}

\author{
Rémi Henriet, ${ }^{1}$ Guoping Lin, ${ }^{1}$ Aurélien Coillet, ${ }^{1,2}$ Maxime Jacquot, ${ }^{1}$ Luca Furfaro, ${ }^{1}$ Laurent Larger, ${ }^{1}$ and \\ Yanne K. Chembo ${ }^{1, *}$ \\ ${ }^{1}$ FEMTO-ST Institute [CNRS UMR6174], Optics Department, 15B Avenue des Montboucons, 25030 Besançon cedex, France \\ ${ }^{2}$ Currently at NIST, Boulder, Colorado 80305, USA \\ *Corresponding author: yanne.chembo@femto-st.fr
}

\begin{abstract}
We report the fabrication for the first time of a strontium fluoride $\left(\mathrm{SrF}_{2}\right)$ whispering-gallery mode resonator with quality factor in excess of 1 billion. The millimeter-size disk-resonator is polished until the surface roughness decreases down to a root-mean square value of $1.2 \mathrm{~nm}$, as measured with a vertical scanning profilometer. We also demonstrate that this ultrahigh $Q$ resonator allows for the generation of a normal-dispersion Kerr optical frequency comb at $1550 \mathrm{~nm}$.
\end{abstract}

Whispering-gallery mode (WGM) resonators have attracted significant attention in recent years, since they provide an interesting platform to explore the nonlinear applications of various optical crystals [1-3]. Among these resonators, mm-size disk-resonators fabricated with mechanical polishing are of particular interest because they can feature ultrahigh $Q$ factors, as high as $\sim 10^{11}$ at $1550 \mathrm{~nm}$ (see Ref. [4]). Another interesting property is that their free spectral range (FSR) is of the order of $10 \mathrm{GHz}$, and this frequency allows for a wide variety of applications in microwave photonics and optical telecommunications.

With the noticeable exception of crystalline quartz [5] only fluorite crystals have allowed for $Q$ factors in excess of $10^{9} \mathrm{in} \mathrm{mm-size} \mathrm{disk} \mathrm{resonators.} \mathrm{These} \mathrm{fluorite} \mathrm{materi-}$ als are namely calcium fluoride $\left(\mathrm{CaF}_{2}\right.$, see $\left.[\underline{4}, \underline{6}-\underline{8}]\right)$, magnesium fluoride $\left(\mathrm{MgF}_{2}\right.$, see [9-11]), and barium fluoride $\left(\mathrm{BaF}_{2}\right.$, see [12]). These crystals belong to the chemical family of metal difluorides, and they share similar optical properties to a large extent. An open point is therefore to investigate if other metal difluorides can feature ultra-high- $Q$ factors as well in the near-infrared frequency range.

In this Letter, we demonstrate for the first time that $\mathrm{SrF}_{2}$ is a metal difluoride that can be used for the fabrication of whispering-gallery mode resonators with $Q$ factors of $10^{9}$ at $1550 \mathrm{~nm}$. The surface profile of the rim has been studied using vertical-scanning optical profilometry, and our fabrication method allows to obtain a surface roughness of $1.2 \mathrm{~nm}$ in root-mean square (rms) value at the rim of the disk. Kerr frequency comb generation in the $\mathrm{SrF}_{2}$ resonator is also reported around $1550 \mathrm{~nm}$, despite the fact that it has a normal material dispersion at this wavelength.

Strontium is an alkaline earth metal located below calcium and above barium in the periodic table, and therefore, the properties of its fluoride counterpart are intermediately between those of $\mathrm{CaF}_{2}$ and $\mathrm{BaF}_{2}$. In particular, $\mathrm{SrF}_{2}$ is a cubic crystal with fluorite structure and lattice constant $0.580 \mathrm{~nm}$, while $\mathrm{CaF}_{2}$ and $\mathrm{BaF}_{2}$ are also both cubic crystals with lattice constants $0.546 \mathrm{~nm}$ and $0.62 \mathrm{~nm}$, respectively [13]. Strontium fluoride has a large transparency window, ranging from $130 \mathrm{~nm}$ to $11 \mu \mathrm{m}$, allowing for a wide variety of optical applications. For instance, its ceramic can be doped with neodymium ions with rather high absorption and emission cross-sections, and a weaker quenching compared to $\mathrm{CaF}_{2}: \mathrm{Nd}^{3+}$ crystals [14]. Such ceramics could have interesting applications for laser and amplifiers in the near-infrared. Similarly to $\mathrm{CaF}_{2}$ and $\mathrm{BaF}_{2}$, the thermo-optic coefficient of strontium fluoride is negative, with a value $d n / d T=12.6 \times$ $10^{-6} \mathrm{~K}^{-1}$, giving it interesting athermal properties.

Related to its crystal structure, and of great interest for the fabrication of resonators, is the hardness of $\mathrm{SrF}_{2}$. Its value is $130 \mathrm{~kg} / \mathrm{mm}^{2}$, a relatively low value compared to other fluoride crystals- $\mathrm{CaF}_{2}$ is $158 \mathrm{~kg} / \mathrm{mm}^{2}$, $\mathrm{MgF}_{2}$ is $415 \mathrm{~kg} / \mathrm{mm}^{2}$, albeit not as low as barium fluoride $\left(82 \mathrm{~kg} / \mathrm{mm}^{2}\right.$ ) [12]. The relative softness of $\mathrm{SrF}_{2}$ allows for a very fast shaping and polishing, while remaining hard enough for our tools and technique to be efficient.

We make a whispering-gallery mode resonator using a grinding and polishing approach [8]. The rim of a commercially available optical window of $\mathrm{SrF}_{2}$ is first shaped into a sharp "V", where the whispering gallery modes will propagate. For that purpose, an air-bearing spindle motor spins the disk, while an abrasive-coated support is brought in contact with the disk. This first step uses silicon carbide powders of large sizes (typically $15 \mu \mathrm{m}$ ) to grind the disk and remove material. Owing to the relative softness of $\mathrm{SrF}_{2}$, this step can be achieved in less than $1 \mathrm{~h}$ when it takes up to $4 \mathrm{~h}$ for a much harder crystal such as magnesium fluoride. The subsequent steps consist in repeating this process with decreasing size of abrasive particles, down to $100 \mathrm{~nm}$.

Once the polishing process is finished, the surface state of the guiding rim is observed using white light vertical-scanning interferometry: the disk is placed on a piezo-controlled stage and under a microscope equipped 

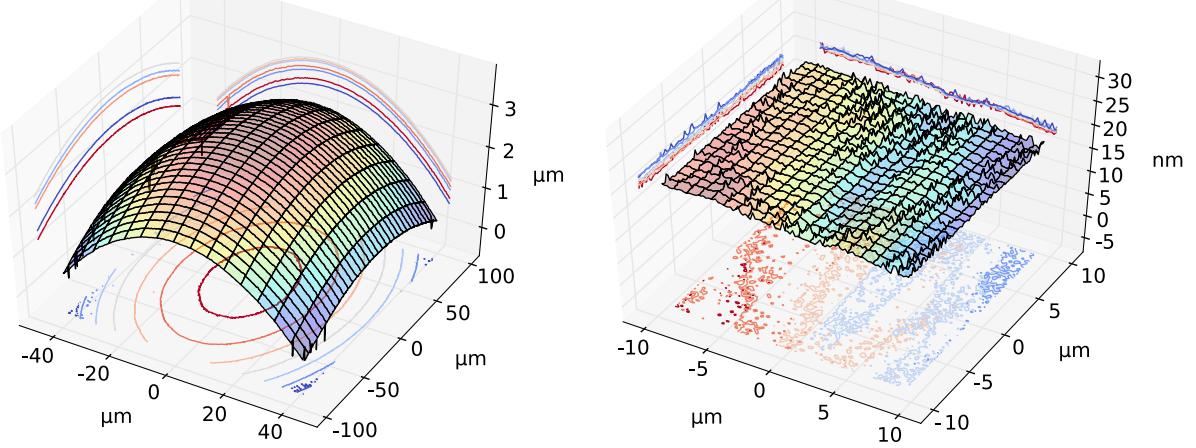

Fig. 1. Profiles of the rim of the disk as measured on a vertical scanning profilometer. In (a), the raw 3D reconstructed surface allows us to measure the major and minor diameter: $D_{\mathrm{M}}=10.6 \mathrm{~mm}$ and $D_{\mathrm{m}}=920 \mu \mathrm{m}$, respectively. This geometry has been corrected on the profile of (b), so that the surface roughness can be measured. The root-mean-square roughness is measured at $R_{\mathrm{RMS}}=1.2 \mathrm{~nm}$.

with a 40× magnification Mirau objective. This setup allows us to reconstruct the local 3D surface profile of the rim of the disk. Such a raw profile is shown on Fig. 1(a) and reveals the expected toroid shape of the whisperinggallery guide. The studied sample has a minor diameter of $D_{\mathrm{m}}=920 \mu \mathrm{m}$ and a major diameter of $D_{\mathrm{M}}=10.6 \mathrm{~mm}$, yielding a free spectral range $\mathrm{FSR}=c /\left(\pi n_{g} D_{\mathrm{M}}\right)=$ $6.3 \mathrm{GHz}$, with $n_{g}=1.43$ being the group velocity index of $\mathrm{SrF}_{2}$ at the telecom wavelength $\lambda=1.55 \mu \mathrm{m}$. By removing the curvature of this toroidal surface, we obtain the corrected profile presented in Fig. 1(b), where we can measure the root-mean-square surface roughness. The measured value is $R_{\mathrm{RMS}}=1.2 \mathrm{~nm}$ in the middle of the studied sample, where the propagating modes will be confined. The roughness increases away from the center of the rim, without influencing the transmission of the nearby optical guide. These measurements show that we can expect very low losses from this resonator. However, this procedure remains a local evaluation of the surface state, and an optical estimate of the quality factor is needed to confirm the good quality factor performance of the resonator.

The cavity ring-down measurement technique is an accurate method to measure the quality factor of an optical resonator, because it is independent of the thermal nonlinearity, and yields both the coupling and intrinsic quality factors [15]. In this method, the light from a continuous-wave laser is coupled to the previously fabricated resonator using the evanescent field of a tapered silica fiber, as shown on Fig. 2. Since the refractive index of $\mathrm{SrF}_{2}(n=1.43$ at the telecom wavelength) is slightly lower than the one of silica, a phase-matching position can be found, and the coupling can be very efficient. The wavelength of the input laser is then quickly swept across a resonance, with a timescale faster than the photon lifetime of the cavity. As a result, the exponential decay of the resonant light interferes with the input light, leading to measurable oscillations. A typical signal observed on the oscilloscope is shown on Fig. 3, where the interferences and exponential decay are clearly visible. By fitting the experimental data with the formula (13) of Ref. [15], one finds that the intrinsic and coupling quality-factors are $Q_{0}=1.04 \times 10^{9}$ and $Q_{e}=1.64 \times 10^{10}$, respectively, yielding a loaded $Q$ factor of $9.76 \times 10^{8}$.
The large value of the coupling $Q$ factor as well as the small transmission dip show that the resonance is strongly undercoupled. This can however be easily overcome by moving the tapered fiber to a slightly smaller diameter, so that the phase-matching condition is better fulfilled.

One can therefore derive an upper bound of the absorption coefficient $\alpha$ of $5.8 \times 10^{-5} \mathrm{~cm}^{-1}$ for $\mathrm{SrF}_{2}$ at $1550 \mathrm{~nm}$, since it is related to the quality factor by $Q=2 \pi n /(\alpha \lambda)$, where $n$ is the refractive index of $\mathrm{SrF}_{2}$ and $\lambda$ is the wavelength. Such very high- $Q$ factors make $\mathrm{SrF}_{2}$ resonators an interesting candidate for nonlinear applications, where large field enhancement at resonance are needed.

WGM resonators are generally used to host enhanced nonlinear photonic interactions, like second-harmonic generation [16-19], or optical hyperparametric interactions (or Kerr comb generation, see [20]). Similarly to most of the fluoride crystals, strontium fluoride offers a wide wavelength range of transparency, from the ultraviolet to the long-wavelength infrared [21]. This property, combined with its low dispersion, makes it a very interesting material for infrared-range systems, and especially for Kerr frequency comb generation. It should be noted

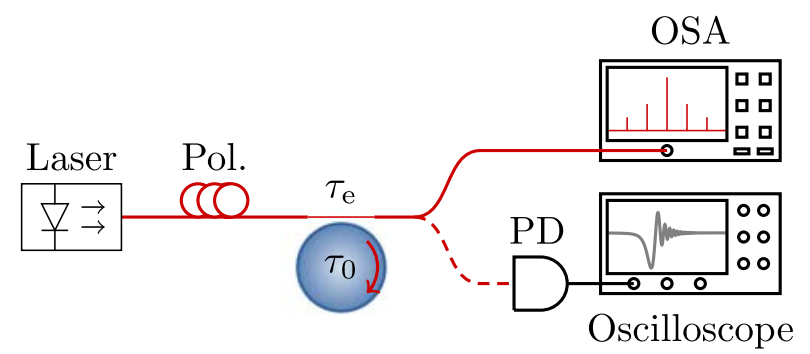

Fig. 2. Experimental setup for the characterization and use of the $\mathrm{SrF}_{2}$ WGM resonator. A continuous-wave laser light is coupled in the resonator via the evanescent coupling of a tapered fiber. For the determination of the $Q$ factor of the disk, the laser frequency is swept, and the time-dependent transmission is detected on a slow photodiode (PD) and recorded on an oscilloscope. In the comb generation experiment, the laser frequency is fixed, and the amplification of the erbium-doped fiber amplifier (EDFA) is increased until a frequency comb is observed on the optical spectrum analyzer (OSA). 


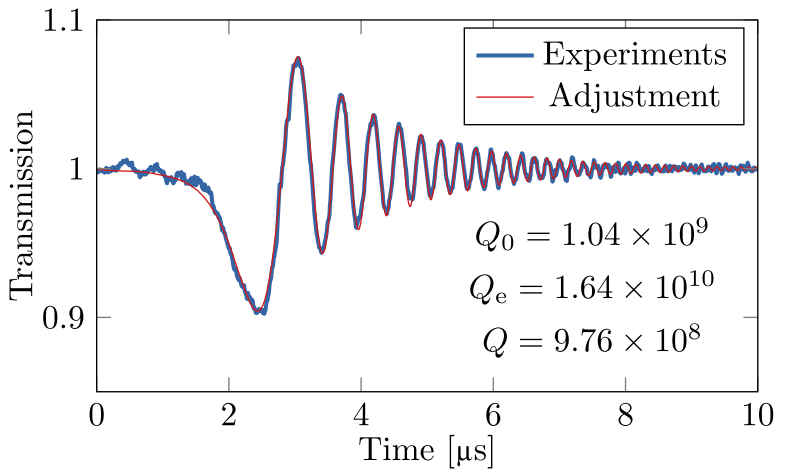

Fig. 3. Transmission of the resonator while the pump laser wavelength is quickly swept over the resonance at the rate of $1.1 \mathrm{MHz} / \mu \mathrm{s}$. This cavity ring-down signal can be fitted with the theoretical expression (red), giving the values for the intrinsic and coupling $Q$ factors: $Q_{0}=1.04 \times 10^{9}$ and $Q_{\mathrm{e}}=1.64 \times 10^{10}$. The loaded $Q$ factor for this resonance is therefore $Q=9.76 \times 10^{8}$.

that Kerr frequency comb generation has already been observed in magnesium fluoride and calcium fluoride WGM resonators. Nevertheless, many other crystalline materials have not yet been explored using the interesting WGM resonator platforms. The zero-dispersion wavelength of $\mathrm{SrF}_{2}$ is at $\lambda=1567 \mathrm{~nm}$ [22], so that the pump wavelength of $\lambda=1550 \mathrm{~nm}$ corresponds to weakly normal group velocity dispersion. The theoretical analysis of Kerr frequency comb generation [23] has shown that the normal regime of dispersion is less favorable for the generation of interesting states, with fewer dynamical states, smaller parameter space regions allowing these states, and more restrictive choice of initial conditions. Nevertheless, we were able to generate frequency combs in our $\mathrm{SrF}_{2}$ disk resonator, as shown in Fig. 4(a). In this experiment, the light of a sub-kHz linewidth continuouswave laser is amplified to few hundreds of milliwatts and coupled in the resonator. Its frequency is tuned to one of the resonances of the disk, and the accurate adjustment of the detuning leads to the generation of a Kerr frequency comb. This comb is similar to other normal combs obtained in calcium fluoride resonators [10], or other normal dispersion materials [24,25], and it can be interpreted as dark or gray solitons circulating inside the cavity.

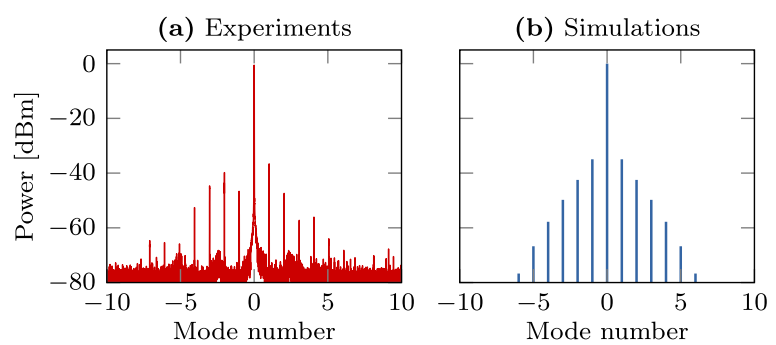

Fig. 4. Optical frequency comb generated in the $\mathrm{SrF}_{2}$ WGM resonator. At a pump wavelength of $1550 \mathrm{~nm}$, the dispersion of the disk is normal $\left(\zeta_{2} / 2 \pi \sim 1 \mathrm{kHz}\right)$, and the generated comb shows similar features as other normal combs [10]. The mode " 0 " is the central (pumped) mode, and the modes with eigennumbers $\pm l$ have a spectral distance $\pm l \times$ FSR with respect to the pump $(\mathrm{FSR}=6.3 \mathrm{GHz})$.
The spectrum displayed in Fig. 4(b) is obtained through the numerical simulation of the dimensionless Lugiato-Lefever [26] equation:

$$
\frac{\partial \psi}{\partial \tau}=-(1+i \alpha) \psi+i|\psi|^{2} \psi-i \frac{\beta}{2} \frac{\partial^{2} \psi}{\partial \theta^{2}}+F,
$$

where $\psi(\theta, \tau)$ is the slowly-varying complex envelope of the intra-cavity field in the co-moving frame, $\theta \in[-\pi, \pi]$ is the azimuthal angle along the circumference, and $\tau=$ $\omega_{\mathrm{C}} t / 2 Q$ is the dimensionless time, with $\omega_{\mathrm{C}}$ being the angular frequency of the resonant cavity mode pumped by the continuous-wave laser at frequency $\omega_{\mathrm{L}}$. The various parameters of this equation are the frequency detuning $\alpha=-2 \sigma / \Delta \omega_{\text {tot }}$ where $\sigma=\omega_{\mathrm{L}}-\omega_{\mathrm{C}}$ is the detuning between the angular frequencies of the pumping laser and the cold-cavity resonance; the overall dispersion parameter $\beta=-2 \zeta_{2} / \Delta \omega_{\text {tot }}$, where $\zeta_{2}$ is the second-order Taylor coefficient of the eigenfrequency expansion; the pump field intensity $F=\left[8 n_{2} c \hbar \omega_{\mathrm{L}}^{2} \Delta \omega_{\text {ext }} / n_{0}^{2} V_{0} \Delta \omega_{\text {tot }}^{3}\right]^{1 / 2}$ $\left[P / \hbar \omega_{\mathrm{L}}\right]^{1 / 2}$ where $P$ is the intensity (in $\mathrm{W}$ ) of the laser pump at the input of the resonator, $n_{0}$ and $n_{2}$ are, respectively, the linear and nonlinear refraction indices of the bulk material, $V_{0}$ is the effective volume of the pumped mode, and $\Delta \omega_{\text {ext }}\left(\Delta \omega_{\text {tot }}\right)$ being the extrinsic (total) modal linewidth $[10,26]$. The various dynamical states arising from this equation have been analyzed in detail in Ref. [23].

In order to obtain the spectrum of Fig. 4(b), the parameters were taken as $\alpha=2.6, \beta=0.4$, and $F^{2}=2.7$, and the initial condition was a negative Gaussian pulse standing on continuous background. It should be noted that while the agreement with the experimental data is good, only a full knowledge of the experimental waveform (including the optical phases) can determine the temporal waveform corresponding to this spectral signature. Nevertheless, the generation of a Kerr frequency comb proves the excellent quality of the $\mathrm{SrF}_{2}$ resonator and its potential for nonlinear applications.

In conclusion, we have reported the fabrication of the first $\mathrm{SrF}_{2}$ whispering-gallery mode resonator with a $10^{9}$ quality-factor using a grinding and polishing approach. The reasonable material softness allows for a very fast fabrication process, and the surface state remains unaffected by the environment, with a roughness of $1.2 \mathrm{~nm}$. The as-fabricated resonator was successfully used for the generation of Kerr frequency combs in the normal regime of dispersion.

The authors acknowledge financial support from the European Research Council (ERC) through the projects NextPhase and Versyt. They also acknowledge financial support from the Center National d'Etudes Spatiales (CNES) through the project SHYRO, from the Région de Franche-Comté, and from the Labex ACTION.

\section{References}

1. A. B. Matsko and V. S. Ilchenko, IEEE J. Sel. Top. Quantum Electron. 12, 3 (2006).

2. V. S. Ilchenko and A. B. Matsko, IEEE J. Sel. Top. Quantum Electron. 12, 15 (2006).

3. A. Chiasera, Y. Dumeige, P. Féron, M. Ferrari, Y. Jestin, G. Nunzi Conti, S. Pelli, S. Soria, and G. C. Righini, Laser Photon. Rev. 4, 457 (2010). 
4. A. A. Savchenkov, A. B. Matsko, V. S. Ilchenko, and L. Maleki, Opt. Express 15, 6768 (2007).

5. V. S. Ilchenko, A. A. Savchenkov, J. Byrd, I. Solomatine, A. B. Matsko, D. Seidel, and L. Maleki, Opt. Lett. 33, 1569 (2008).

6. A. A. Savchenkov, A. B. Matsko, D. Strekalov, M. Mohageg, V. S. Ilchenko, and L. Maleki, Phys. Rev. Lett. 93, 243905 (2004).

7. I. S. Grudinin, N. Yu, and L. Maleki, Opt. Lett. 34, 878 (2009).

8. A. Coillet, R. Henriet, K. Phan Huy, M. Jacquot, L. Furfaro, I. Balakireva, L. Larger, and Y. K. Chembo, J. Vis. Exp. 78, e50423 (2013).

9. W. Liang, A. A. Savchenkov, A. B. Matsko, V. S. Ilchenko, D. Seidel, and L. Maleki, Opt. Lett. 36, 2290 (2011).

10. A. Coillet, I. Balakireva, R. Henriet, K. Saleh, L. Larger, J. Dudley, C. Menyuk, and Y. Chembo, IEEE Photon. J. 5, 6100409 (2013).

11. A. Coillet and Y. K. Chembo, Opt. Lett. 39, 1529 (2014).

12. G. Lin, S. Diallo, R. Henriet, M. Jacquot, and Y. K. Chembo, Opt. Lett. 39, 6009 (2014).

13. M. Weber, Handbook of Optical Materials, Laser \& Optical Science \& Technology (Taylor \& Francis, 2002).

14. T. T. Basiev, M. E. Doroshenko, V. A. Konyushkin, and V. V. Osiko, Opt. Lett. 35, 4009 (2010).

15. Y. Dumeige, S. Trebaol, L. Ghisa, T. K. Ngan Nguyen, H. Tavernier, and P. Feron, J. Opt. Soc. Am. B 25, 2073 (2008).
16. V. S. Ilchenko, A. A. Savchenkov, A. B. Matsko, and L. Maleki, Phys. Rev. Lett. 92, 043903 (2004).

17. J. Fürst, D. Strekalov, D. Elser, M. Lassen, U. L. Andersen, C. Marquardt, and G. Leuchs, Phys. Rev. Lett. 104, 153901 (2010).

18. G. Lin and N. Yu, Opt. Express 22, 557 (2014).

19. G. Lin, J. U. Fürst, D. V. Strekalov, and N. Yu, Appl. Phys. Lett. 103, 181107 (2013).

20. T. J. Kippenberg, R. Holzwarth, and S. A. Diddams, Science 332, 555 (2011).

21. E. Palik, Handbook of Optical Constants of Solids, (Elsevier Science, 1985), Vol. 1.

22. A. Feldman, D. Horowitz, R. M. Waxler, and M. J. Dodge, "Optical characterization final technical report," Tech. Rep. 993, (National Bureau of Standards, 1979).

23. C. Godey, I. V. Balakireva, A. Coillet, and Y. K. Chembo, Phys. Rev. A 89, 063814 (2014).

24. X. Xue, Y. Xuan, Y. Liu, P. Wang, S. Chen, J. Wang, D. E. Leaird, M. Qi, and A. M. Weiner, "Mode interaction aided soft excitation of dark solitons in normal dispersion microresonators and offset-frequency tunable kerr combs," arXiv:1404.2865 (2014).

25. W. Liang, A. A. Savchenkov, V. S. Ilchenko, D. Eliyahu, D. Seidel, A. B. Matsko, and L. Maleki, Opt. Lett. 39, 2920 (2014).

26. Y. K. Chembo and C. R. Menyuk, Phys. Rev. A 87, 053852 (2013). 